\title{
Essai critique sur la diffusion numérique dans un algorithme d'écoulement transitoire à surface libre
}

\author{
C. Thirriot, L. Benayada \\ Institut de mécanique des fluides \\ Institut national polytechnique de Toulouse
}

\section{Introduction}

Ce qui suit est en quelque sorte un hommage à Alexandre PrEISSMANN qui fut dès la fin des années cinquante à la SOGREAH un des pionniers enthousiastes et imaginatifs du calcul informatique au service de l'hydraulique. En parallèle avec l'expérience menée à la SOGREAH mais tout à fait indépendamment, à Toulouse en 1959, l'un des auteurs expérimentait un des premiers programmes de calcul numérique d'intumescences (C. THIRRIOT [19]). II avait la surprise de voir apparaître des ondulations secondaires en front d'onde alors que les accélérations verticales, cause physique d'un tel phénomène physique, n'étaient pas prises en compte dans le modèle mathématique. C'était le premier contact avec les artefacts numériques qui peuvent fallacieusement donner l'illusion de la réalité naturelle. Mais souvent plus que les oscillations parasites stationnaires dont les ondulations secondaires sont un rare cas d'analogie, c'est la diffusion numérique qui se glisse subrepticement dans les algorithmes et s'étale dans les résultats.

Cette diffusion numérique est la raison cachée du succès de la fameuse méthode graphique proposée par le célèbre hydraulicien italien G. DE MARCHI en 1945 [7] pour le suivi des crues dans une rivière. L'algorithme sous-jacent au procédé graphique n'était qu'un enrichissement non linéaire de la fameuse méthode de Muskingum proposée vers 1935 par Mac Carthy et le Geological
Survey Service pour le calcul de la propagation des crues avec application à la rivière Muskingum.

Jusqu'en 1960, il ne semble pas que les hydrauliciens qui se servaient de ces méthodes aient nettement perçu que l'étalement apparent des crues provenait de la diffusion numérique née de la discrétisation des variables d'espace et de temps. Ensuite les études critiques ont proliféré. A l'aide de la transformée de Laplace, l'étude littérale de l'équation aux différences permet d'obtenir la solution analytique du schéma de Muskingum (C. THIR. RIOT [21]), mais la mise en évidence la plus approfondie du caractère diffusif de ce schéma est certainement l'étude proposée par J. CungE en 1969 [4] dans le Journal de Recherche Hydraulique. Ceci a été si bien reconnu par l'opinion hydraulicienne internationale que l'on parle maintenant de la méthode de Muskingum-Cunge.

Mais le schéma à quatre points utilisé dans cette méthode n'est qu'un aspect simplifié de celui employé par A. PREISSMANN à SOGREAH [13] dans l'algorithme implicite de résolution des équations de Saint Venant, algorithme qui lui aussi, fait intervenir de la diffusion numérique, diffusion qui est préjudiciable à la bonne représentation de phénomènes à variation rapide comme les intumescences.

L'appréciation de cette diffusion numérique parasite est plutôt délicate par manque de référence. Souvent, on procède par comparaison avec des résultats obtenus pour de'minuscules pas de temps et d'espace où le caractère

\section{Critical test about numerical diffusion of transient flow algorithm with free surface}

In this paper, a critical study of the numerical diffusion associated to an hyperbolic-type operator governing transient free surface flow, is presented. The numerical diffusion is estimated through the discretisation of the simplified governing equations, using finite differences implicit scheme. The diffusion is due to the choice of the space and time steps when using implicit scheme. The interpretation of the results is made using the existing similarity with a simple oscillating system. 
hyperbolique de l'opérateur continu est assez bien conservé dans la transcription numérique mais ceci coûte extrêmement en temps de calcul et l'on n'est jamais parfaitement sûr du passage à la limite. Des études analytiques fines des schémas numériques ont cependant été développées au cours des dernières décennies (A. Daubert [6], J.A. Liggett [8] et M. Ponce [10]).

Mais dans ce qui suit, nous allons suivre un cheminement différent, tout à fait empirique fondé sur l'observation des résultats de simulation numérique. Nous nous intéressons à un cas particulier : caractérisé par l'absence de perte de charge et de pente du fond. L'écoulement transitoire considéré est celui provoqué par une brusque variation de vitesse à l'amont d'un bief de canal débouchant à l'aval dans un réservoir à niveau constant. Le profil en travers du canal est supposé rectangulaire mais l'on aurait pu tout aussi bien considérer un profil trapézoïdal sans complication aucune. L'énorme avantage de ce cas simplifié, c'est que l'on connaît la solution théorique et exacte fournie de manière extrêmement simple par la théorie des caractéristiques.

Pour garder à la présentation des résultats un caractère très concret, nous n'avons pas voulu faire l'étude en grandeurs adimensionnelles et nous n'avons considéré qu'un seul cas d'écoulement. Mais procédant par induction, on verra que l'on peut en tirer fructueusement un enseignement assez général.

\section{Construction de la solution numérique de l'écoulement transitoire}

\subsection{Préliminaire et hypothèses}

Nous choisirons donc d'étudier la propagation et la réflexion d'intumescences à front raide. Ce faisant, nous savons qu'en considérant un front raide, nous faisons une entorse à la réalité car en fait il s'agit soit de ressaut mobile bouillonnant, soit d'ondulation secondaire accompagnant le front. Mais dans les équations de BARRÉ DE SAINT VENANT, ne sont pas prises en compte les accélérations verticales qui vont expliquer les ondulations secondaires. La théorie des caractéristiques dans le cas d'un écoulement idéal sans perte de charge dans un canal sans pente fait apparaître la propagation et la réflexion de discontinuités brusques comme l'indiquent les schémas des figures 1 à 4 .

Pour ne pas avoir des scrupules à propos du déferlement ou carambolage des ondes superficielles, nous avons considéré des variations très modérées du débit (mais variations brusques) ce qui nous permettrait de considérer éventuellement l'hypothèse de quasi-linéarité de l'opérateur aux dérivées partielles.

Notre objectif est la diffusion numérique à l'état pur d'où l'élimination de l'effet de perte de charge. Nous laissons de côté l'étude des algorithmes numériques, nous les prenons en praticien, globalement, et nous regarderons leurs effets sur deux aspects : vitesse à l'aval et hauteur d'eau à amont.
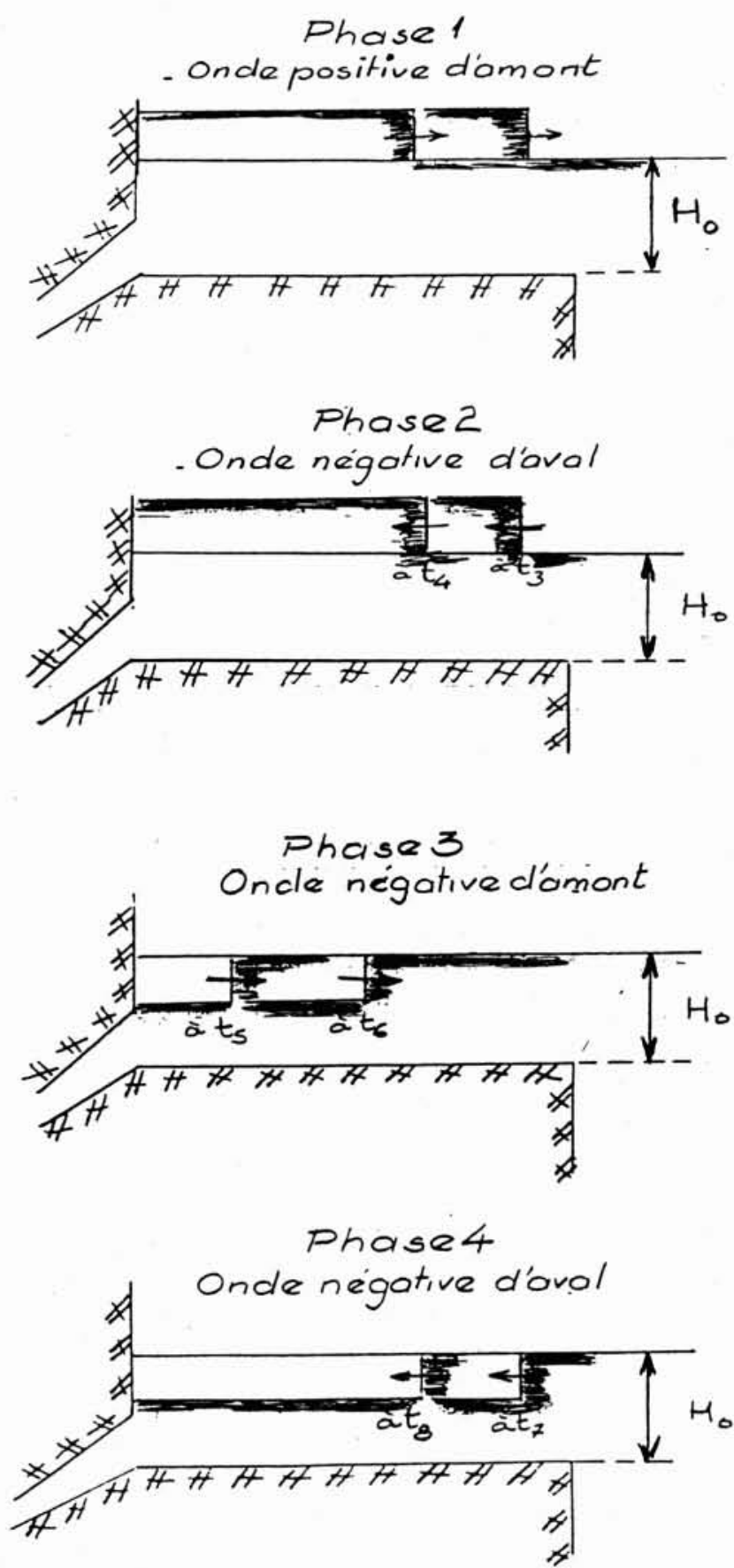

1 à 4. Propagation et réflexion d'intumescences à front raide dans un canal sans pente.

\subsection{Equations et notations}

Les équations de l'écoulement transitoire à surface libre sont celles, classiques, de BARRÉ De SAINT VENANT (J.J. STOKER [17], STRElKofF [18]). Pour la suite, nous introduirons les écarts $h$ et $v$ par rapport aux valeurs de la hauteur $H_{0}$ et de vitesse $V_{0}$, en régime permanent antérieur uniforme.

$$
\begin{aligned}
& H=H_{0}+h \\
& V=V_{0}+v .
\end{aligned}
$$


De plus, nous ferons les hypothèses suivantes :

$$
\frac{h}{H_{0}} \ll 1 \quad \text { et } \quad \frac{v}{V_{0}} \ll 1 .
$$

Dans cet article nous nous limiterons au cas où la pente du fond est nulle et les pertes de charge négligeables, ainsi la forme linéarisée des équations de SAINT VENANT peut se réduire à ce qui suit :

$$
\begin{aligned}
& \frac{\partial h}{\partial t}+V_{0} \frac{\partial h}{\partial x}+H_{0} \frac{\partial v}{\partial x}=0 \\
& \frac{\partial v}{\partial t}+V_{0} \frac{\partial v}{\partial x}+g \frac{\partial h}{\partial x}=0 .
\end{aligned}
$$

\subsection{Conditions initiales et aux frontières}

L'écoulement initial est uniforme. En ce qui concerne les deux conditions aux frontières amont et aval nécessaires pour que le problème soit bien posé en écoulement fluvial, nous avons choisi de fixer la hauteur constante à l'aval (ce qui peut correspondre grosso modo à un débouché dans un réservoir) et de considérer les effets d'une augmentation brusque $v_{0}$ de la vitesse moyenne dans la section amont (ce qui entraînera une augmentation de débit légèrement modulée par la variation de hauteur).

\subsection{Solution théorique}

La plupart des lecteurs de la Houille Blanche savent bien que la théorie des caractéristiques permet d'obtenir la solution exacte correspondant à cet écoulement idéal avec ces conditions aux frontières simples. Nous ne reviendrons pas sur l'obtention de cette solution mais nous en rappellerons brièvement le comportement. La brusque variation de vitesse à l'amont induit une onde positive d'amont marquée par une surélévation brusque qui se propage de manière conservative jusqu'à l'aval (phase 1 , fig. 1). A l'aval, la condition de niveau constant provoque la réflexion d'onde qui physiquement se traduit par la vidange de l'eau accumulée en surélévation avec un débit quasi double de celui qui continue d'être injecté à l'amont (phase 2, fig. 2). Cette onde négative d'aval arrivant à l'amont se réfléchit en poursuivant la vidange et donc en maintenant un débit vers l'aval supérieur au débit injecté à l'amont (phase 3, fig. 3). Arrivée à l'aval, après avoir créé un creux dans tout le bief, cette onde négative se réfléchit et se transforme en onde de remplissage qui efface le creux de volume par le fait d'une quasi annulation du débit dans la section aval (phase 4, fig. 4). Cette onde positive d'aval arrivant à l'amont en s'y réfléchissant double son effet de surélévation en reconstituant la surélévation produite à l'instant initial par l'injection brusque du débit provoquant le supplément de vitesse supposé maintenu par la suite. Le cycle est ainsi fermé et le phénomène recommence identique pour une nouvelle période avec les quatre phases décrites ci-dessus. Avec l'hypothèse de phénomène conservatif sans dissipation d'énergie due aux pertes de charge et sans effet de la gravité dû à la pente, théoriquement il y a reproduction perpétuelle de ce cycle de manière périodique.

Dans la réalité physique, évidemment, les évolutions ne sont plus idéales. Mais on peut visualiser assez bien les allers et retours d'onde dans un canal de laboratoire. Seulement, du fait de la variation de la célérité des ondes superficielles avec la hauteur d'eau, les ondes positives font apparaître un déferlement et les ondes négatives, un étalement des fronts d'onde.

La solution théorique montre donc un perpétuel régime transitoire avec passage de valeurs minimales à valeurs maximales avec valeurs intermédiaires égales à la vitesse amont ou à la hauteur aval, valeurs qui sont maintenues constantes entre deux passages de front d'onde raide positive ou négative.

\subsection{Solution numérique}

Dans la littérature, on trouve différents modèles numériques, mais on s'intéressera particulièrement au schéma numérique implicite proposé par PREISSMANN [13] qui est largement utilisé pour la prévision de la propagation des ondes de crues sur les rivières à fond fixe. Ce schéma à quatre points centré dans l'espace et décentré dans le temps, généralisé ultérieurement par GoodwiNg [9] a même été employé pour la prévision dans le cas où le fond est mobile par CORREIA [3], RAHUEL [15] et YANG [24].

Dans le plan $(x, t)$ de la figure 5 , entre deux instants $n \Delta t$ et $(n+1) \Delta t$ et entre les sections $j \Delta x$ et $(j+1) \Delta x$, pour toute fonction continue dérivable, PREISSMANN a proposé les approximations suivantes pour les dérivées :

$$
\begin{gathered}
\frac{\partial f}{\partial x}=\frac{1}{\Delta x}\left[\theta\left(f_{j+1}^{n+1}-f_{j}^{n+1}\right)+(1-\theta)\left(f_{j+1}^{n}-f_{j}^{n}\right)\right] \\
\frac{\partial f}{\partial t}=\frac{1}{2 \Delta t}\left[\left(f_{j+1}^{n+1}-f_{j+1}^{n}\right)+\left(f_{j}^{n+1}-f_{j}^{n}\right)\right]
\end{gathered}
$$

$\theta$ est un coefficient de pondération.

Ainsi, à chaque temps $n \Delta t$ on aboutit à un système linéaire de la forme:

$$
\begin{aligned}
& a_{1} v_{j}^{n+1}+a_{2} h_{j}^{n+1}+a_{3} v_{j+1}^{n+1}+a_{4} h_{j+1}^{n+1}=g_{1}^{k} \\
& b_{1} v_{j}^{n+1}+b_{2} h_{j}^{n+1}+b_{3} v_{j+1}^{n+1}+b_{4} h_{j+1}^{n+1}=g_{2}^{k}
\end{aligned}
$$

où : $a_{i}, b_{i}, g_{1}$ et $g_{2}$ dépendent de différentes caractéristiques, à savoir :

- caractéristiques numérique $(\theta, \Delta t, \Delta x)$

- caractéristiques de l'écoulement initial $\left(V_{0}, H_{0}\right)$

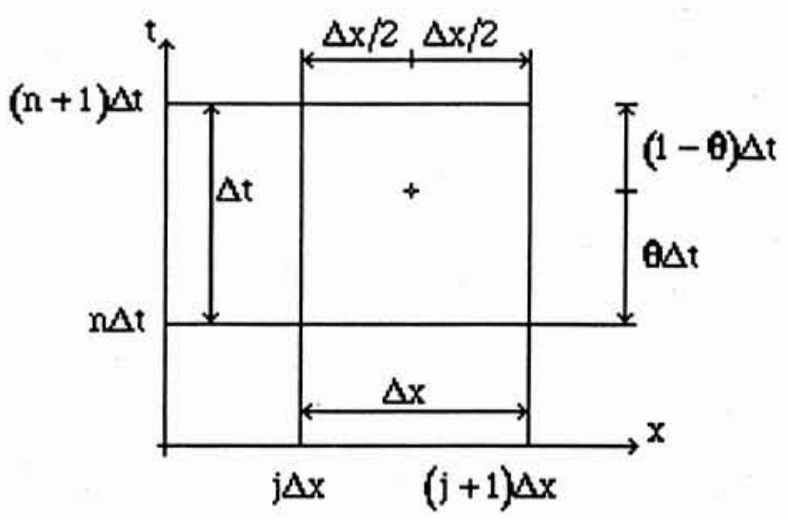

5. Schématisation de discrétisation dans le plan $(x, t)$. 
— caractéristiques de l'écoulement à l'instant antérieur $n \Delta t$.

L'ensemble des équations ainsi obtenues sera fermé par l'utilisation des conditions aux frontières suivantes :

$$
\begin{aligned}
& V(0, t)=V_{0}+v_{0} \Rightarrow v(0, t)=v_{0} \\
& H(L, t)=H_{0} \quad \Rightarrow h(L, t)=0 .
\end{aligned}
$$

\subsection{Bref aperçu sur l'étude théorique des propriétés de l'algorithme}

Comme nous l'avons déjà indiqué, plusieurs chercheurs se sont employés à cerner par l'étude analytique les comportements des algorithmes numériques utilisés en hydrodynamique. Les cheminements peuvent paraître différents à première vue mais à y regarder de plus près, l'analyse consiste toujours à regarder suivant l'approche traditionnelle de VON NEUMANN, comment évolue dans le temps et dans l'espace une perturbation de la forme $e^{\omega t+4 x}$, $\omega$ et $\varphi$ étant des nombres complexes. Si on focalise l'atténuation sur l'évolution dans le temps, à une abscisse donnée, après avoir posé $\omega=\omega_{1}+i \omega_{2}$, pour que le comportement de l'algorithme soit stable il faut que $\omega_{1}$ soit négatif ou nul puisque l'amplitude de la perturbation évoluera comme $e^{\omega_{1} \prime}$.

Comment détermine-t-on les paramètres $\omega$ et $\varphi$ ? Par l'étude des valeurs propres de l'opérateur associé à l'algorithme numérique. En effet, il faut bien voir que la perturbation de la solution numérique dont on parle ne pourra proliférer que si elle correspond à une condition de résonance, résonance du fondamental ou d'un harmonique. Et l'on sait que les valeurs propres sont les paramètres caractéristiques des modes de résonance.

Cette façon de voir et de faire revient encore à considérer que la solution du système d'équations aux différences associé par discrétisation au système d'équations aux dérivées partielles originelles, peut s'écrire sous la forme d'un développement en série dans l'espace des fonctions propres (ou vecteurs propres) associées aux valeurs propres dont la forme la plus simple et bien connue est le développement en série de Fourier associé à la solution théorique du problème simple que nous nous proposons d'examiner.

Pour revenir plus précisément à notre propos qui est l'évolution de la solution numérique de l'algorithme de Preissmann, nous voyons donc que l'essentiel est de déterminer les éléments des valeurs propres caractérisés par $\omega$, pulsation complexe, et $\varphi$, nombre d'onde, en s'attachant plus particulièrement à la partie réelle $\omega_{1}$ de $\omega$ et ceci en fonction des découpages $\Delta x$ et $\Delta t$.

L'idée est simple : on reporte dans les équations aux différences homogènes la solution indéfinie $e^{\omega t+\varphi x}$ et on recherche les couples $(\omega, \varphi)$ pour lesquels le système aux dérivées admet une solution non triviale (différente de zéro).

L'idée est simple, certes, mais le processus calculatoire est un peu laborieux. Nous ne le détaillerons pas ici, nous renvoyons les lecteurs intéressés aux articles de CORREIA [3], Liggett [8] et Ponce [10].

Dans sa thèse, Correia lorsqu'il présente les conditions de stabilité du schéma de PREISSMANN généralisé, insiste sur l'importance du sens de déplacement des ondes marqué par le nombre de courant $C_{r}$. Il aboutit à une formule donnant l'atténuation en fonction de $\theta$, $c_{r}$ et $\Delta x / L$ et du décentrage dans l'espace repéré par le paramètre $\phi$.

Après Liggett et Cunge [8], Ponce et al. [10] approfondissent l'étude théorique de la stabilité du schéma à quatre points, centré en $x$, en tenant compte de l'effet d'une perte de charge linéarisée. Ils mettent aussi en évidence l'expression de la célérité numérique et de la différence entre les coefficients d'atténuation (décréments logarithmiques) afférents à la solution numérique et au comportement physique de base et ceci seulement pour le terme fondamental, mais étant entendu que la procédure peut être appliquée aux harmoniques. Les résultats issus de calculs enchaînés foisonnants ne paraissent exploitables qu'à l'aide d'abaques (qui semblent construits pour un nombre d'onde imposé).

Nous voudrions aussi signaler une approche particulièrement élégante proposée par A. DAUBERT qui, de manière alerte et lumineuse, met en évidence la construction des valeurs propres du schéma de Preissmann et propose de manière très originale leur représentation graphique dans le plan complexe comme résultat d'une transformation conforme simple qu'est l'inversion. Une présentation particulièrement séduisante qui est un régal pour l'esprit face aux cheminements de fantassin traditionnels.

Comme notre propos d'aujourd'hui n'est pas dans le délayage des approches analytiques antérieures mais dans une approche directe empirique sans prérequis mathématique, à la portée de tous, nous allons maintenant brosser le contexte de notre expérience.

\section{Exemple d'application}

\subsection{Données}

Comme nous l'avons déjà annoncé, pour conserver un caractère très concret à l'étude critique, nous avons choisi de parler d'un exemple simple en considérant les valeurs physiques avec leurs unités plutôt que d'utiliser des valeurs adimensionnelles, certes plus générales, mais aussi plus abstraites.

Les caractéristiques de l'exemple sont les suivantes :

- longueur du canal :

$L=1000 \mathrm{~m}$

— vitesse en régime permanent initial :

$V_{0}=0.5 \mathrm{~m} / \mathrm{s}$

— hauteur en régime permanent initial : $H_{0}=2 \mathrm{~m}$

- conditions aux frontières :

$$
\begin{array}{ll}
\text { limite amont } & v(0, t)=0,1 \mathrm{~m} / \mathrm{s} \\
\text { limite aval } & H(L, t)=2 \mathrm{~m}
\end{array}
$$

- en ce qui concerne le coefficient de pondération, comme cela a été fait ultérieurement par plusieurs auteurs [6], si $\theta \in] 1 / 2,1$ [ alors le schéma est inconditionnellement stable. Par la suite, nous avons choisi de fixer ce coefficient à 0,8 .

\subsection{Caractéristiques de l'écoulement}

Calculons quelques paramètres caractéristiques tels que le nombre de Froude initial et la période. La célérité propre en écoulement initial est :

$$
C_{0}=\sqrt{g H_{0}}=4,43 \mathrm{~m} / \mathrm{s}
$$


Tableau 1. - Caractéristiques des essais de simulation.

\begin{tabular}{|c|c|c|c|c|c|c|c|c|c|c|}
\hline$\Delta\{s]$ & 25 & 50 & 100 & 200 & 375 & 750 & 1500 & 3000 & 4500 & 6000 \\
\hline$\Delta x[m]$ & 50 & 100 & 200 & 500 & 1000 \\
\hline$\Delta t_{0}(\Delta x)[s]$ & 11,3 & 22,6 & 45,2 & 112,1 & 225,3 \\
\hline
\end{tabular}

d'où le nombre de Froude de l'écoulement porteur

$$
F_{0}=V_{0} / C_{0}=0.5 / 4.43=0.113 .
$$

Ce nombre de Froude est faible. La période qui est la durée de deux allers et retours d'onde est, pour une faible perturbation

$$
\begin{aligned}
& T_{0}=2\left(\frac{L}{C_{0}+V_{0}}+\frac{L}{C_{0}-V_{0}}\right) \# \\
& \# 4 \frac{L}{C_{0}\left(1-F_{0}^{2}\right)}=915 \mathrm{s.}
\end{aligned}
$$

La durée de la simulation la plus utilisée $(T=3000 \mathrm{~s})$ permet de suivre le phénomène pendant un peu plus de trois périodes.

\subsection{Caractéristiques des essais numériques}

Les diverses valeurs utilisées pour les pas de temps et d'espace sont indiquées dans le tableau 1.

A titre indicatif pour chaque $\Delta x$ nous avons aussi présenté les durées de propagation $\Delta t_{0}$ (moyennes des temps de propagation d'onde amont et d'onde aval).

Comme les couples $(\Delta x, \Delta t)$ utilisés dans le calcul numérique couvrent toutes les possibilités d'association, on voit que généralement l'onde numérique (caractérisée par la vitesse fictive $\Delta x / \Delta t$ ) marche moins vite que l'onde physique ce qui correspond aux circonstances habituelles d'emploi des méthodes implicites où l'on souhaite diminuer le volume de calcul.

\section{Etude critique}

\subsection{Remarques générales}

A partir des différents essais de simulations, il ressort les constats suivants :

- si l'on fait varier uniquement le pas de distance $\Delta x$, en gros, il y a deux zones. Une première correspondant au faible pas où pratiquement ce dernier $n$ 'influe pas et une seconde où il est d'une importance majeure; en effet au-delà d'un certain pas, il y a un déphasage considérable entre la solution de référence et la solution numérique ;

- si maintenant le pas de temps $\Delta t$ varie seul, il y a aussi deux zones mais cette fois la première zone correspond au faible pas de temps où le modèle mathématique est reproduit avec un amortissement parasite dans le temps. Dans la seconde zone de $\Delta t$ très fort, la réponse semble tendre directement, du fait d'un amortissement très fort, vers la solution asymptotique du régime permanent qui s'établirait s'il y avait perte de charge physique.

\subsection{Résultats}

Avant de pousser plus loin l'analyse, il faut remarquer que le phénomène de diffusion qui se traduit ici par des oscillations amorties (apparemment sinusoïdales pour $\Delta t$ modéré) apparaît très vite, même pour des valeurs de $\Delta t$ faible fraction de la période propre du phénomène physique. Par exemple, on pourrait croire que prendre $\Delta t=25 \mathrm{~s}$ (qui correspond presque à un quarantième de la période) assure un découpage suffisamment fin pour respecter l'aspect physique du phénomène. Eh bien non, l'atténuation de la fonction créneau est déjà forte au bout de quelques allers et retours.

Pour ne pas surcharger les figures et les multiplier, nous avons choisi de représenter seulement deux situations symptomatiques : l'évolution de la vitesse au débouché du canal (fig. 6) et celle du tirant d'eau dans la section d'entrée (fig. 7) sur une durée de simulation de $3000 \mathrm{~s}$ pour les pas $\Delta x \leqslant 750 \mathrm{~s}$. Ainsi, il est possible de suivre l'évolution du phénomène pendant plus de trois périodes. Pour les très grands pas de temps, la simulation a évidemment été faite sur une durée supérieure à $3000 \mathrm{~s}$.

La représentation des graphes numériques est un peu difficile à lire mais nous n'avons pas voulu relier les points successifs afin de laisser aux graphes tout leur caractère discret évidemment de plus en plus sensible lorsque le pas de temps $\Delta t$ augmente. 


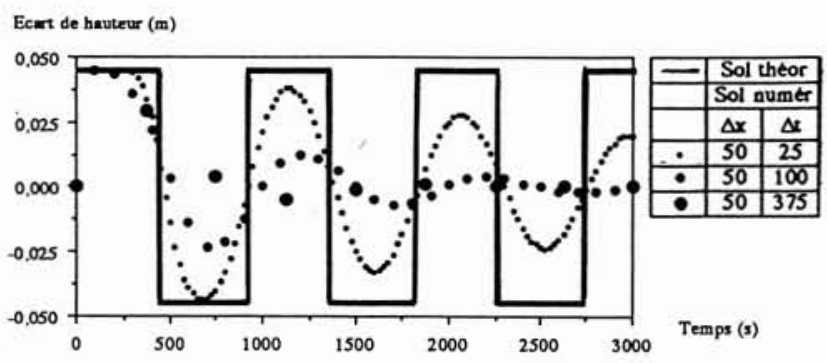

7. Comparaison entre solutions analytique et numérique pour la hauteur amont.

\subsection{Analyse}

A partir des résultats de simulation, il faut admettre l'importance de l'amortissement de la solution numérique. Afin de quantifier cet amortissement et vu l'allure des courbes, l'idée est de faire une analogie entre les résultats du système d'équations aux dérivées partielles et ceux de l'équation différentielle linéaire homogène du second ordre à coefficients constants de la forme :

$$
\frac{\mathrm{d}^{2} y}{\mathrm{~d} t^{2}}+2 \mu \frac{\mathrm{d} y}{\mathrm{~d} t}+\omega_{0}^{2} y=0
$$

dont la solution classique est la suivante :

$$
y(t)=C e^{-\mu t} \sin (\omega t+\varphi)
$$

avec :

$$
\begin{array}{ll}
\mu & : \text { coefficient d'amortissement } \\
\omega_{0} & : \text { pulsation propre } \\
\omega & : \text { pulsation }\left(\omega^{2}=\omega_{0}^{2}-\mu^{2}\right) \\
C \text { et } \varphi \quad: \text { constante et déphasage dépendant des condi- } & \text { tions initiales. }
\end{array}
$$

Cette équation différentielle est par exemple, en hydraulique, celle qui gère les oscillations dans un système d'amenée galerie-cheminée d'équilibre. Nous insistons bien sur le fait qu'il ne s'agit que d'analogie suggérée par la ressemblance des graphes. Ce sera pour nous un moyen commode pour synthétiser l'analyse des résultats numériques. C'est une constatation inductive tirée de l'observation de la réalité expérimentale numérique et nous ne chercherons pas pour l'instant un semblant de justification par exemple dans la dégénérescence des deux équations aux dérivées partielles en un système différentiel après seule discrétisation par rapport au temps (opération utilisée par l'un de nous il y a une trentaine d'années (C. ThirRiot, H. BARTHet [1968]). Dans notre cas, il sera procédé à un calage de la vitesse et de la hauteur par des fonctions de la forme ci-dessous :

$$
\begin{aligned}
& h(L, t)=C_{1 h}+C_{2 h} e^{-\mu_{h} t} \sin \left(\omega_{h} t+\varphi_{h}\right) \\
& v(0, t)=C_{1 v}+C_{2 v} e^{-\mu_{v} t} \sin \left(\omega_{v} t+\varphi_{v}\right)
\end{aligned}
$$

A priori l'ajustement dépend de 10 paramètres, c'est beaucoup de degrés de liberté. Mais, si l'on veut que l'analyse ait un caractère concret et physique, on est amené à poser que $\omega_{h}=\omega_{v}$ et $\mu_{h}=\mu_{v}$, c'est-à-dire à admettre que l'on retrouve les mêmes modes propres dans les évolutions des deux grandeurs associées $v$ et $h$. En fait nous laisserons d'abord flotter l'indépendance entre $\mu_{h}$ et $\mu_{v}$ aussi bien par curiosité du résultat que par souci de simplicité de la mise en œuvre. Mais après mise à l'échelle convenable des fluctuations de vitesse et de niveau, nous avons pu, par simple translation, superposer parfaitement les graphes $v(t)$ et $h(t)$ obtenus numériquement figures 8 et 9 , ce qui prouve bien que les équations aux différences linéaires, obtenues pour $v$ et $h$, et base du calcul numérique, admettent les mêmes valeurs propres. Par ailleurs, nous espérons que le lissage indiquera que les termes $C_{1 h}$ et $C_{1 v}$ sont égaux aux valeurs asymptotiques, car dans le cas contraire, ils seraient la preuve d'un biais introduit par l'algorithme numérique.

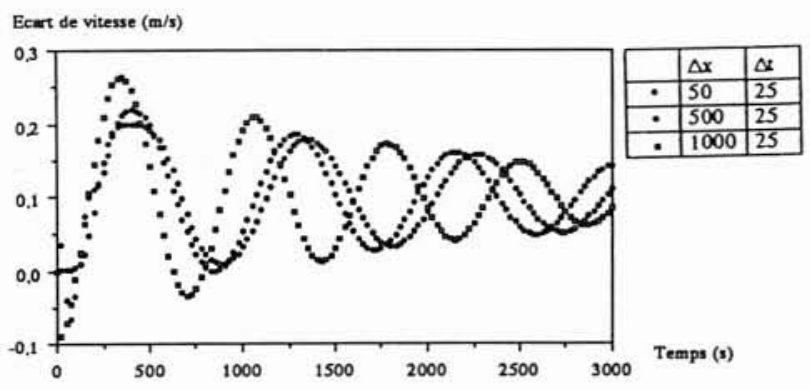

8. Comparaison entre solutions numériques pour la vitesse aval.

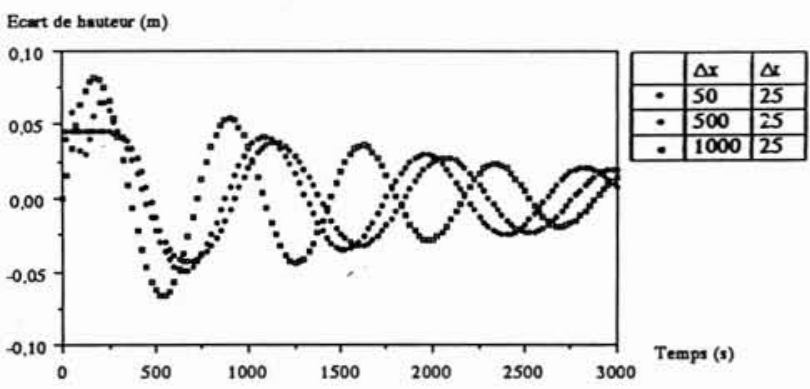

9. Comparaison entre solutions numériques pour la hauteur amont.

Le calage est a priori une opération non linéaire. En fait, tant que l'amortissement n'est pas exagéré et qu'il y a assez de points pour déceler les extremums avec précision, la procédure est grandement simplifiée si l'on examine l'évolution en fonction du temps des amplitudes d'oscillation en ordonnée logarithmique, pour la vitesse au débouché aval $(x=L)$ et la hauteur dans la section d'entrée.

Les figures 10 et 11 montrent les résultats pour un pas d'espace $\Delta x=50 \mathrm{~m}$ et différents pas de temps. La bonne linéarité des graphes empiriques résultant de l'anamorphose des résultats numériques, confirme notre choix intuitif d'une analogie de comportement de système simple oscillant. Le phénomène imposé par le découpage 
est déterministe, mais le caractère discret des résultats en $t$ laisse un résidu chaotique qui explique le léger aspect aléatoire des résultats synthétiques.

A partir du résultat de lissage des graphes analogues à ceux des figures 10 et $I I$, nous avons pu dresser les tableaux donnant les coefficients d'amortissement apparents empiriques afférents aux différents pas d'espace et de temps (tabl. 2 et 3 ).

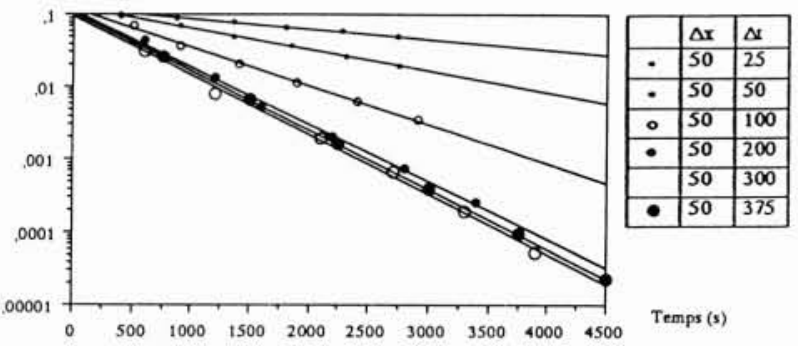

10. Comparaison des amplitudes des vitessés numériques pour différents pas $\Delta t$.

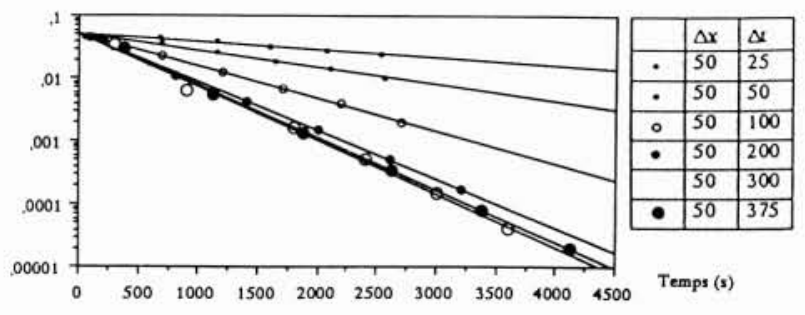

11. Comparaison des amplitudes des vitesses numériques pour différents pas $\Delta t$.

Il est bien évident que nous ne prétendons pas significatifs tous les chiffres figurant dans les tableaux. Mais nous avons voulu donner les résultats bruts de l'ajustement suivant la méthode des moindres carrés nous réservant de jouer sur l'incertitude pour homogénéiser ensuite les résultats. Nous pensons que, grosso modo, deux chiffres significatifs doivent suffire pour caractériser $\mu$ le coefficient de diffusivité (ou viscosité) apparent.

Que peut-on déduire à l'examen de ces tableaux ?

Tableau 2. - Valeurs des amortissements numériques de la vitesse $\left(\mu_{n} \times 10^{4}\right)$.

\begin{tabular}{|c|c|c|c|c|c|}
\hline 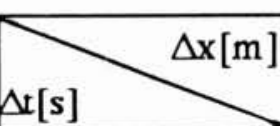 & 50 & 100 & 200 & 500 & 1000 \\
\hline 25 & 3,15 & 3,14 & 3,26 & 3,86 & 5,75 \\
\hline 50 & 6,80 & 6,78 & 6,79 & 7,50 & 11,0 \\
\hline 100 & 12,3 & 12,0 & 12,1 & 13,3 & 18,9 \\
\hline 200 & 18,1 & 18,1 & 17,6 & 19,6 & 23,6 \\
\hline 300 & 18,9 & 18,9 & 18,9 & 19,5 & 24,1 \\
\hline 375 & 18,9 & 18,9 & 19,0 & 20,1 & 22,7 \\
\hline 500 & 18,5 & 18,5 & 18,4 & 17,6 & 20,3 \\
\hline 750 & 14,5 & 14,4 & 14,5 & 15,0 & 16,7 \\
\hline 1500 & 8,77 & 8,77 & 8,76 & 8,79 & 8,89 \\
\hline 3000 & 3,20 & 3,20 & 3,21 & 3,19 & 3,12 \\
\hline 4500 & 2,26 & 2,24 & 2,24 & 2,24 & 2,24 \\
\hline 6000 & 1,78 & 1,75 & 1,78 & 1,75 & 1,75 \\
\hline
\end{tabular}

Tableau 3. - Valeurs des amortissements numériques de la hauteur $\left(\mu_{n} \times 10^{4}\right)$.

\begin{tabular}{|c|c|c|c|c|c|}
\hline$\Delta \times[\mathbf{s}]$ & 50 & 100 & 200 & 500 & 1000 \\
\hline 25 & & & & & \\
\hline 50 & 2,86 & 2,89 & 2,87 & 4,27 & 5,71 \\
\hline 100 & 11,9 & 6,25 & 6,37 & 7,63 & 11,1 \\
\hline 200 & 17,8 & 12,0 & 12,0 & 12,9 & 18,6 \\
\hline 300 & 19,5 & 17,8 & 18,0 & 19,8 & 24,9 \\
\hline 375 & 19,2 & 19,5 & 19,5 & 19,8 & 23,9 \\
\hline 500 & 17,5 & 19,2 & 19,3 & 20,0 & 22,9 \\
\hline 750 & 15,0 & 17,6 & 17,7 & 19,3 & 20,2 \\
\hline 1500 & 8,86 & 15,0 & 15,0 & 14,8 & 15,80 \\
\hline 3000 & 5,64 & 8,86 & 8,86 & 8,87 & 9,89 \\
\hline 4500 & 3,29 & 5,64 & 5,62 & 5,52 & 5,25 \\
\hline 6000 & 2,39 & 3,29 & 3,29 & 3,28 & 3,23 \\
\hline & & 2,39 & 2,39 & 2,39 & 2,37 \\
\hline
\end{tabular}


- le pas $\Delta x$ semble avoir peu d'influence sur la force de l'amortissement (sauf lorsqu'il atteint toute la longueur du canal) ;

- une légère différence apparaît entre le coefficient d'amortissement de la vitesse à l'aval et celui de la hauteur à l'amont (qui est plus faible en moyenne d'environ 3 à $10 \%$ ). La différence serait plus forte pour les faibles valeurs de $\Delta t$ (par exemple pour $\Delta t=25 \mathrm{~s}$ ). Cette constatation doit nous inciter à la prudence dans la généralisation mais grosso modo, avec un certain flou, un coefficient approximatif suffit à caractériser à la fois les évolutions de la vitesse et de la hauteur ;

- l'influence du pas de temps $\Delta t$ est décisive sur le coefficient d'amortissement.

\subsection{Evolution pour des pas de temps $\Delta t$ faibles ou modérés}

Pour rendre plus concrète l'évolution du coefficient équivalent d'amortissement, nous avons représenté le graphe $(\mu, \Delta t)$ sur la figure 12 en prenant pour $\mu$ une valeur moyenne pour $\Delta x<500 \mathrm{~m}$.

Cette illustration suggère que l'évolution de $\mu$ en fonction de $\Delta t$ est d'abord grossièrement linéaire pour $\Delta t<100 \mathrm{~s}$. Dans cette zone linéaire du graphe, le coefficient d'amortissement (ou décrément logarithmique) serait approximativement :

$$
\begin{aligned}
\Delta t<100 \mathrm{~s}, \quad \mu=\frac{\Delta t}{T^{* 2}}=1,23 \cdot 10^{-5} \Delta t, & \text { avec } \\
T^{*} & \approx 283 \mathrm{~s} .
\end{aligned}
$$

14

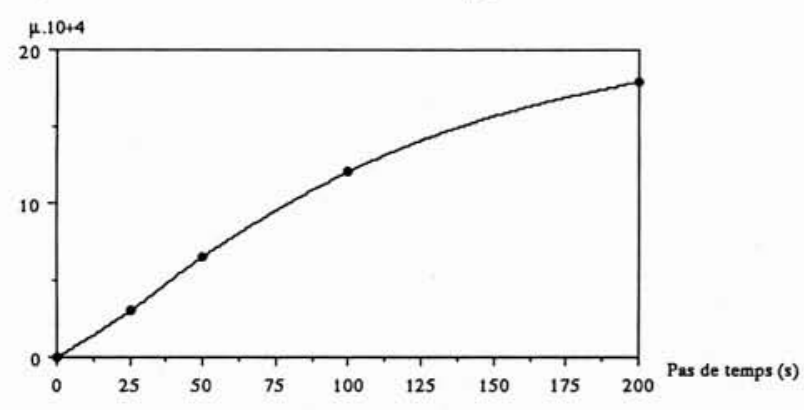

12. Evolution de $\mu$ en fonction du pas $\Delta t$.
Enfin l'infléchissement du graphe $(\mu, \Delta t)$ pour $\Delta t$ supérieur à $100 \mathrm{~s}$, incite à profiter plus à fond de l'analogie avec le système oscillant amorti. En effet, cette analogie a déjà été pour nous fort fructueuse. Bien sûr, ceci ne constitue pas une preuve ou un fondement de validité. Mais l'on peut envisager d'aller plus loin dans l'analogie en pensant aux circonstances d'amortissement critique (avec la disparition des oscillations) et d'amortissement apériodique.

\subsection{Recherche empirique sur l'amortissement numérique critique}

Pour guider l'approche empirique, rappelons les résultats classiques des systèmes oscillants amortis, pivot de notre analogie. La période de l'oscillation devient infinie, la pulsation disparaît si $\mu=\omega_{0}, \omega_{0}$ étant la fréquence propre du système non amorti. Ici a priori, on pourrait penser que $\omega_{0}$ correspond à la période des ondes soit $T_{0} \# 915 \mathrm{~s}$ et $\omega_{0}=6,87 \cdot 10^{-3}$. Alors :

$$
\omega=\sqrt{\omega_{0}^{2}-\mu^{2}} .
$$

Au-delà de $\mu=\omega_{0}$, l'évolution serait purement exponentielle construite sur les deux fonctions propres:

$$
e^{-\left(\mu+\sqrt{\left.\mu^{2}-\omega_{0}^{2}\right)}\right.} \text { et } e^{-\left(\mu-\sqrt{\left.\mu^{2}-\omega_{0}^{2}\right)}\right.} \text {. }
$$

Voyons si dans l'analogie, on peut tirer un avantage pour la description du comportement numérique des intumescences. L'explication est plus délicate pour la pulsation que celle faite pour le coefficient d'amortissement dans le cas des $\Delta t$ modérés. En effet, on dispose de peu de points par période tant que l'oscillation existe et même si l'on admet la réalisation parfaite de l'analogie il y aura difficulté à déterminer les paramètres parce que les points disponibles ne constituent pas les extremums. L'identification des paramètres est alors un processus non linéaire et on est obligé de travailler par itérations.

Auparavant nous avons essayé d'éprouver la transposition de la relation liant $\omega$ et $\mu$ pour les valeurs des $\Delta t$ modérés. Ceci conduit au tableau 4.

La confrontation entre valeur empirique $\mu_{\mathrm{e}}$ et valeur théorique $\mu_{t}=\sqrt{\omega_{0}^{2}-\omega_{\text {app }}^{2}}$ n'est pas fameuse. Mais il faut dire que la procédure d'identification de $\omega$ est délicate et peu précise. Pour $\Delta t$ petit, une très faible variation de $\omega_{\text {app }}$ entraîne une très forte variation de $\mu_{r}$. Aussi dans un souci critique avons-nous pris l'algo-

Tableau 4. - Récapitulatif des résultats.

\begin{tabular}{|c|c|c|c|c|c|c|}
\hline$\Delta t[\mathrm{~s}]$ & 25 & 100 & 300 & 500 & 750 & 1500 \\
\hline $\mathrm{T}_{\text {app }}[\mathrm{s}]$ & 930 & 1010 & 1320 & 1700 & 2100 & 3600 \\
\hline$\omega_{\text {app }} \times 10^{3}$ & 6,8 & 6,2 & 4,8 & 3,7 & 3,0 & 1,7 \\
\hline$\omega_{\mathrm{t}} \times 10^{3}$ & 0,98 & 2,96 & 4,91 & 5,79 & 6,18 & 6,66 \\
\hline$\mu_{\mathrm{t}} \times 10^{3}$ & 6,86 & 6,76 & 6,60 & 6,63 & 6,71 & 6,81 \\
\hline$\mu_{\mathrm{e}} \times 10^{3}$ & 0,30 & 1,20 & 1,92 & 1,80 & 1,47 & 0,88 \\
\hline$\mu_{\mathrm{t}} / \mu_{\mathrm{e}}$ & 3,27 & 2,47 & 2,55 & 3,22 & 4,20 & 7,57 \\
\hline
\end{tabular}




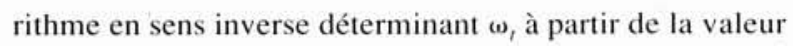
observée $\mu_{r}$. L'écart entre les valeurs $\omega_{\text {app }}$ et $\omega$, est tel qu'objectivement on doit conclure sportivement que l'analogie ne peut être prolongée aussi loin. Certes $\omega_{\text {app }}$ décroît lorsque $\mu_{\mathrm{c}}$ augmente mais en fait bien plus vite que ne le voudrait l'analogie. Le coefficient d'amortissement entraînant le régime apériodique serait inférieur à la valeur théorique critique $\mu_{r}=6,87 \cdot 10^{-3}$.

\subsection{Examen des circonstances d'amortissement apériodique}

\subsubsection{Présentation des faits numériques}

Dans cette partie, il est question du cas où la réponse tend rapidement vers la solution asymptotique. Comme auparavant, on se limite à la représentation des mêmes situations asymptotiques, soit la vitesse aval (fig. 13) et la hauteur amont (fig. 14).

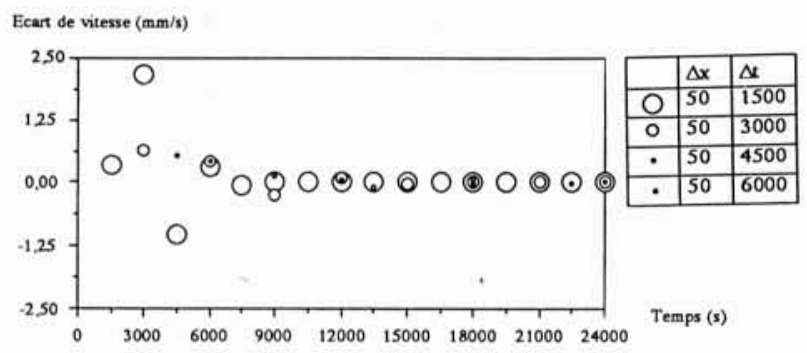

13 Comparaison entre solutions numériques pour la vitesse aval.

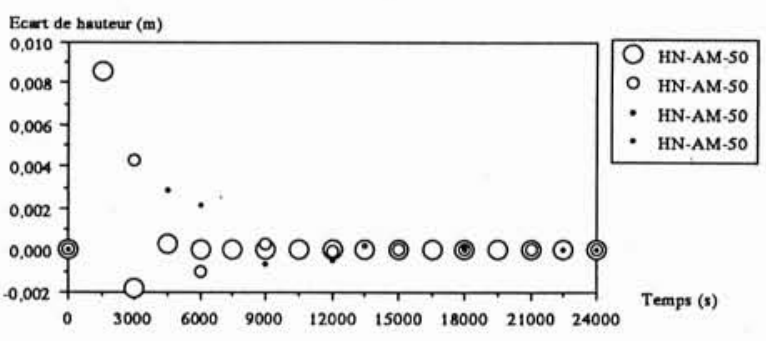

14. Comparaison entre solutions numériquess pour la hauteur amont.

Nous notons qu'il n'y a pas de régime vraiment monotone apériodique. Ainsi, il faut souligner un léger dépassement de la valeur asymptotique ou même une alternance infime autour de celle-ci. L'alternance est peut-être due au bruit de l'arrondi. Mais nous resterons prudents dans notre induction, on ne peut exclure que ce léger frémissement résiduel soit inhérent au caractère oscillatoire irréductible de l'opérateur de discrétisation. Mais, il n'en reste pas moins que vu globalement pour l'interprétation physique, l'amortissement apparaît comme apériodique.

\subsubsection{Résultats empiriques de l'amortissement numérique}

Les résultats synthétiques, valeurs numériques du coefficient apparent d'amortissement numérique, ont déjà été présentés dans les tableaux 2 et 3 où l'on voit des valeurs du paramètre $\Delta t$ allant bien au-delà de l'inflexion du graphe $\mu(\Delta t)$ à s'éloigner de l'évolution linéaire. Ce qui frappe pour les grandes valeurs de $\Delta t$, c'est la décroissance indubitable du coefficient $\mu$ d'amortissement apparent lorsque $\Delta t$ croît. Ce fait empirique nous a d'abord désarçonnés puis une fois de plus nous sommes revenus à l'analogie du système oscillant. En effet lorsque l'évolution d'un tel système devient apériodique, c'est que les valeurs propres sont réelles telles que :

$$
\begin{aligned}
& \mu_{1}=\mu+\sqrt{\mu^{2}-\omega_{0}^{2}} \\
& \mu_{2}=\mu-\sqrt{\mu^{2}-\omega_{0}^{2}} .
\end{aligned}
$$

La solution est alors de la forme

$$
y=C_{1} \mathrm{e}^{-\mu_{1} t}+C_{2} \mathrm{e}^{-\mu_{2} t} .
$$

Les valeurs $\mu_{1}$ et $\mu_{2}$ sont très vite contrastées lorsque $\Delta t$ s'éloigne en croissant de la valeur critique $\Delta t_{c}$ telle que $\mu_{c}=\omega_{0}$.

$$
\begin{gathered}
\frac{\mu_{1}}{\mu_{2}}=\frac{\left(\mu+\sqrt{\mu^{2}-\omega_{0}^{2}}\right)^{2}}{\omega_{0}^{2}} . \\
\text { Si } \quad \mu \gg \omega_{0} \text { alors, } \frac{\mu_{1}}{\mu_{2}} \approx\left(\frac{2 \mu}{\omega_{0}}\right)^{2} .
\end{gathered}
$$

Ceci explique que très vite dans la « solution » analogique donnant $y$ en fonction de $t$, le premier terme disparaisse. Supposant que la partie principale de $y$ soit

$$
y_{p}=C_{2} \mathrm{e}^{-\mu_{2} t} .
$$

Que devient $\mu_{2}$ lorsque $\mu$ augmente ?

$$
\mu_{2}=\mu-\sqrt{\mu^{2}-\omega_{0}^{2}}=\frac{\omega_{0}^{2}}{\mu+\sqrt{\mu^{2}-\omega_{0}^{2}}} .
$$

Ainsi donc $\mu_{2}$ qui devient l'amortissement apparent diminue si $\mu$, valeur qui serait intrinsèquement liée à $\Delta t$, augmente. Ceci est en très bon accord avec ce qu'on aperçoit sur les lissages des résultats numériques. Cette constatation redonne un regain d'intérêt à notre analogie.

\subsubsection{Caractérisation du dépassement de la valeur asymptotique}

Puisque cette analogie de système oscillant est si fructueuse, pourquoi ne pas chercher à l'exploiter encore pour tenter d'expliquer les dépassements de la valeur asymptotique observée en régime apériodique ? Revenons à la solution analogique :

$$
y=C_{1} \cdot \mathrm{e}^{-\mu_{1} \cdot t}+C_{2} \cdot \mathrm{e}^{-\mu_{2} \cdot t} .
$$


Cette solution permet en effet l'apparition d'un maximum.

$$
\text { Si } \frac{\mathrm{d} y}{\mathrm{~d} t}=0, \quad C_{1} \mu_{1} \mathrm{e}^{-\mu_{1} t_{m}}+C_{2} \mu_{2} \mathrm{e}^{-\mu_{2} t_{m}}=0
$$

alors

$$
\begin{aligned}
y\left(t_{m}\right)=C_{2} \mathrm{e}^{-\mu_{2} t_{m}}(1+ & \left.\frac{C_{1}}{C_{2}} \mathrm{e}^{-\left(\mu_{1}-\mu_{2}\right) t_{m}}\right)= \\
& =C_{2} \mathrm{e}^{-\mu_{2} t_{m}}\left(1-\frac{\mu_{2}}{\mu_{1}}\right) .
\end{aligned}
$$

Comme

$$
\left(\mu_{1}-\mu_{2}\right) t_{m}=\log \left(-\frac{C_{1} \mu_{1}}{C_{2} \mu_{2}}\right),
$$

il suffit que $C_{1}$ et $C_{2}$ soient de signes différents pour que la circonstance d'un maximum au-dessus de la valeur asymptotique soit possible. Autre conséquence, après ce maximum il n'y aura qu'une évolution monotone. Des oscillations constatées ne seraient plus qu'un bruit numériquement engendré par les erreurs d'arrondi, mais encore une fois il s'agit là d'une hypothèse seulement suggérée par l'induction.

\subsection{Graphe de synthèse}

Notre objectif était de caractériser l'amortissement. Nous proposons donc à titre de synthèse dans la figure 15, les graphes $\mu_{e}(\Delta t)$ relatifs à la vitesse et à la hauteur qui sont tracés à partir du calcul de la moyenne de $\mu_{e}$ pour $\Delta x \leqslant 200 \mathrm{~m}$. Il est à noter la bonne concordance entre les deux graphes, sauf pour le point correspondant à $\Delta t=3000 \mathrm{~s}$ mais à notre avis cette différence n'est que le résultat d'un artefact numérique lors de l'évaluation de $\mu_{e}$.

\section{Conclusion}

Notre propos était de montrer sur un cas concret de manière simple et empirique mais indubitable, l'effet de la diffusion artificielle numérique dans l'étude de la propagation des intumescences qui sont un cas exacerbé d'écoulement transitoire à surface libre. Comme il y a toujours des germes d'irréversibilités dans les phénomènes naturels, on s'accommode souvent assez bien de résultats numériques faisant apparaître des atténuations d'amplitude. Et pourtant celles-ci peuvent n'être qu'un artefact de calcul comme le montre l'examen d'un cas théorique à perte de charge nulle. La stabilité est certes assurée par les algorithmes implicites mais le souci d'économie du temps d'ordinateur peut conduire en prenant des pas de temps trop grands à dénaturer la reproduction du modèle physique.

L'analogie des systèmes oscillants, sans rien prouver, permet de mettre en évidence des paramètres synthétiques afférant d'une part à l'anamorphose et d'autre part à l'allongement de la période du phénomène jusqu'à l'installation d'un régime apériodique si l'on prend des pas de temps trop grands.

On peut considérer que la synthèse de nos expériences numériques est faite avec le graphe de la figure 15 qui donne l'évolution du coefficient d'amortissement apparent $\mu_{e}$ en fonction de son facteur essentiel, le pas de temps $\Delta t$. Deux zones sont à distinguer. Dans une première, $\mu_{e}$ croît avec $\Delta t$ jusqu'à un maximum où $\Delta t$ correspond approximativement au tiers de la période propre du phénomène de propagation ; les graphes numériques sont alors du type oscillant amorti. Dans la deuxième zone, le coefficient d'amortissement apparent $\mu_{e}$ décroît et les graphes de résultats numériques deviennent apériodiques.

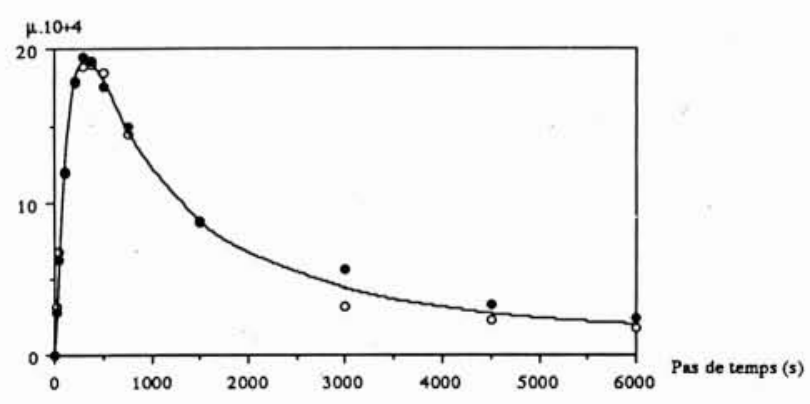

15. Evolution de $\mu$ en fonction du pas $\Delta t$.

Le pas d'espace $\Delta x$ joue un rôle surtout lorsqu'il devient très grand, au moins de l'ordre de la moitié de la longueur du canal. Alors il provoque une ondulation parasite déferlant au-delà du créneau qui représente la réponse théorique fournie par la théorie des caractéristiques. L'étude numérique, à titre pédagogique, a été menée sur un seul cas d'écoulement. Il n'est pas question d'effectuer un examen exhaustif pour toute la plage de variation du paramètre essentiel $\theta$ de l'algorithme de Preissmann. La lourdeur de simulations numériques accumulées ne résiste pas devant la généralité des résultats des études analytiques soigneuses lorsqu'il s'agit de systèmes linéaires. Mais la simulation numérique prend l'avantage lorsqu'on examine des opérateurs non linéaires, en particulier dans les conditions aux limites, qui alors ne permettent plus de bénéficier de la convolution.

Encore une fois, nous redisons que notre propos ne s'adressait pas à un aréopage de numériciens avertis mais voulait être une mise en garde concrète et pratique des praticiens de l'hydraulique qui utilisent de plus en plus des logiciels clé en main. Et bien que toute notre illustration ait été faite sur le schéma de Preissmann ils peuvent augurer avec nous, que des comportements similaires peuvent apparaître avec des schémas encore implicites mais plus riches et à plus forte convergence. 


\section{Bibliographie}

(1) ANGEL N.M., RUBEN N. (1985), - Wave attenuation in open channel flow. Encyclopedia of Fluids Mechanics. Vol. 2, Dynamics of Single-Fluid Flows and Mixing, chapter 7. 174-202.

[2] BeNAYADA L. (1991). - Approche théorique et comparaison avec solution numérique des équations de Saint-Venant. Rapport interne $N^{*} 2$. Institut de Mécanique des Fluides, Toulouse.

[3] CORRE1A L.R.P. (1990), - Numerical modeling of unsteady sediment transport. Thèse présentée pour l'obtention du grade de Docteur ès-sciences de l'Ecole Polytechnique Fédérale de Lausanne.

[4] Cunge J.A. (1969). - On the subject of a flood propagation computation method (Muskingum method). I. Hydr. Res.. $7(2), 205-230$.

[5] DAUBERT A. (1964). - Quelques aspects de la propagation des crues, La Houille Blanche. N*3, 341-346.

[6] DALBERT A. (1993). - Quelques considérations sur l'étude numérique des équations de Saint-Venant. Correspondance particulière. mai.

[7] DE MARChI G. (1945), - Sull'onda di piena che seguirebbe al crollo della diga di cancano. L'Energia Elettrica. Vol. XXII, Nor 8-9-10. Milano, 157-169.

|8| LiggetT J.A.. Cung J.A. (1975), - Numerical methods of solution of the unsteady flow equations. Unsteady flow in open channel, Edited by K. Mahmood and V. Yevjevich, Water resources publications, Fort Collins, Colorado, USA. Vol. I. 89-182.

19] LyN D.A., Goobwin P. (1987), - Stability of a general Preissmann scheme. I. Hydr. Engrg., ASCE, 112, 16-28.

$110]$ PONCE V.M., INDLEKOFER H. and Simons D.B. (1978). - Convergence of four-point implicit water wave models. I. Hydr. Dis... ASCE, 104(7). 947-958.

III] Ponce V.M. CheN Y.H. and Simons D.B. (1979). - Unconditional stability in convection computations. J. Hydr. Div.. ASCE, 105(9). 1079-1086.

[12] Ponce V.M., Theurer F.D. (1982), - Accuracy Criteria in Diffusion Routing. I. Hydr. Div.. ASCE, 180(6), 1982. 747-757.
113] Preissmann A. (1961). - Propagation des intumescences dans les canaux et rivières. Premier Congrès de l'Association Française de Calcul. Grenoble, septembre.

114] Preissmann A. (1972). - Numerische uerfahren zur berechnung instationärer gerinneströmunger. LehrgangsSymposium. Elektronische berechnung Von rohr-und gerinneströmunger nürnberg. Oktober.

[15] RAhuel J.L. (1988), - Modélisation de l'évolution du lit des rivières alluvionnaires à granulométrie étendue. Thèse présentée pour l'obtention du titre de Docteur de l'Université Joseph Fourier. Grenoble I.

[16] Sмгтн G.D. (1979). - Numerical solution of partial differential equations : finite difference methods. Second edition. Oxford Applied Mathematics and Computing Science Series. 75-141.

[17] STOKER J.L. (1957), - Water Waves. Intersciences publishers, Inc, New York, N.Y.

|181 StrelkofF T. (1969). - One dimensional equations of open channel flow, I. Hydr. Div., ASCE, 861-876.

[19] Thirriot C. (1959). - Sur le phénomène de propagation d'une onde de choc dans une galerie de fuite d'usine hydrotechnique. C.R.A.S., tome 249, p. 2716-2718.

[20| Thirriot C. (1960), - Contribution à l'étude des régimes variés et des régimes transitoires dans les canaux d'usines. Thèse présentée à la faculté des Sciences de l'Université de Toulouse, pour obtenir le grade de Docteur ès-sciences Physiques, $\mathrm{N}^{*}$ d’Ordre: 1262, décembre.

[21] Thirriot C.. Barthet H. (1968), - Possibilité et limite de la dégénérescence en système différentiel du modèle mathématique des intumescences. La Houille Blanche $\mathrm{N}^{*} 7$.

[22] Thirriot C. (1968). - Critique d'un algorithme de calcul de propagation des crues. C.R.A.S., tome 266, p. 90.

[23] Thirriot C. (1968). - Comparaison de méthodes de calcul de propagation des ondes de crue. $\mathrm{X}^{\mathrm{e}}$ Journées de la Société Hydrotechnique de France, question 2, rapport 10, juin. Paris.

[24] YANG J.L. (1989), - Modélisation de l'évolution du lit des rivières alluvionnaires à granulométrie étendue. Thèse présentée pour l'obtention du titre de Docteur de l'Université Joseph Fourier, Grenoble I. 